\title{
Autonomic nervous system: a key player in prodromal multiple sclerosis?
}

\author{
Mario Habek $^{1,2} \cdot$ Magdalena Krbot Skorić ${ }^{1,3}$
}

Received: 26 February 2020 / Accepted: 27 February 2020 / Published online: 11 March 2020

(c) Springer-Verlag GmbH Germany, part of Springer Nature 2020

Multiple sclerosis (MS) is a complex inflammatory autoimmune and neurodegenerative disorder affecting individuals between 20 and 40 years of age, more often women. The incidence and prevalence of MS are increasing globally, also in geographic areas that were previously considered to be low incidence for the disease [1]. Given the cumulative number of patients with MS worldwide and the increasing disease-modifying therapies becoming available, early diagnosis is key, as early intervention may arrest disease progression [2].

Several lines of evidence suggest that the pathophysiological processes of MS begin years before the onset of obvious clinical signs or symptoms, in what it is referred to as presymptomatic or prodromal MS. Should individuals with MS in the prodromal phase could be reliably identified, treatment in this early phase could potentially prevent further manifestations of the disorder.

The first line of evidence supporting prodromal MS is the existence of asymptomatic, otherwise healthy individuals with neuroimaging evidence of CNS demyelination consistent with MS, a condition referred to as radiologically isolated syndrome (RIS) [3]. Approximately a third of these asymptomatic individuals will develop clinically definite MS, within an average time of 5 years.

The second line of evidence is based on studies performed with data obtained from electronic health records and administrative data claim showing an increase in the number of physician visits, hospital admissions, and drug prescriptions in the 5 years before the first demyelinating

Mario Habek

mhabek@mef.hr

1 Department of Neurology, Referral Center for Autonomic Nervous System Disorders, University Hospital Center Zagreb, Kišpatićeva 12, 10000 Zagreb, Croatia

2 School of Medicine, University of Zagreb, Zagreb, Croatia

3 Faculty of Electrical Engineering and Computing, University of Zagreb, Zagreb, Croatia event in subjects who were eventually diagnosed with MS $[4,5]$. Two studies further aimed to identify the symptoms for which people who will later in life develop MS sought medical attention in the period up to 14 years before being diagnosed with MS. In the first study, the authors found that individuals with MS in the period up to 10 years prior to first MS symptom, suffer from four groups of symptoms: autonomic, pain, cognitive/fatigue, and psychiatric symptoms. Within the autonomic group, upper gastrointestinal symptoms (e.g. nausea and vomiting), urinary dysfunction (e.g. incontinence and retention), and anorectal symptoms were the most prevalent ones [6]. Another important finding from this study is that more symptomatic burden was associated with increased risk for MS, with a $51 \%, 29 \%$, and $20 \%$ increased risk of MS for each additional symptom at 0-2, $2-5$, and 5-10 years before the diagnosis date, respectively [6].

In another retrospective analysis, in a cohort of patients with MS, 122 patients had gastrointestinal symptoms on average 3.7 years before the first clinical manifestation of MS [7]. In this cohort, $50 \%$ of patients had constipation, $29.5 \%$ had diarrhea, $17.2 \%$ had irritable bowel syndrome, and $3.3 \%$ had fecal incontinence.

Finally, a study which investigated serum neurofilament light chain (sNfL), a marker of axonal damage, showed that sNfL levels were significantly higher compared to matched controls up to 6 years prior to the diagnosis of MS [8]. This study has provided objective evidence of neuroaxonal degeneration several years prior to development of first MS symptom, further characterizing the prodromal MS phase.

Autonomic nervous system abnormalities are being increasingly recognized as an important aspect of MS at the clinical, neurophysiological, and molecular level. Although the evidence is still very preliminary, autonomic dysfunction in patients with MS appears to follow a distinctive pattern: the disease activity (manifested with clinical relapses and brain MRI demyelinating activity) is associated with sympathetic nervous system dysfunction, 


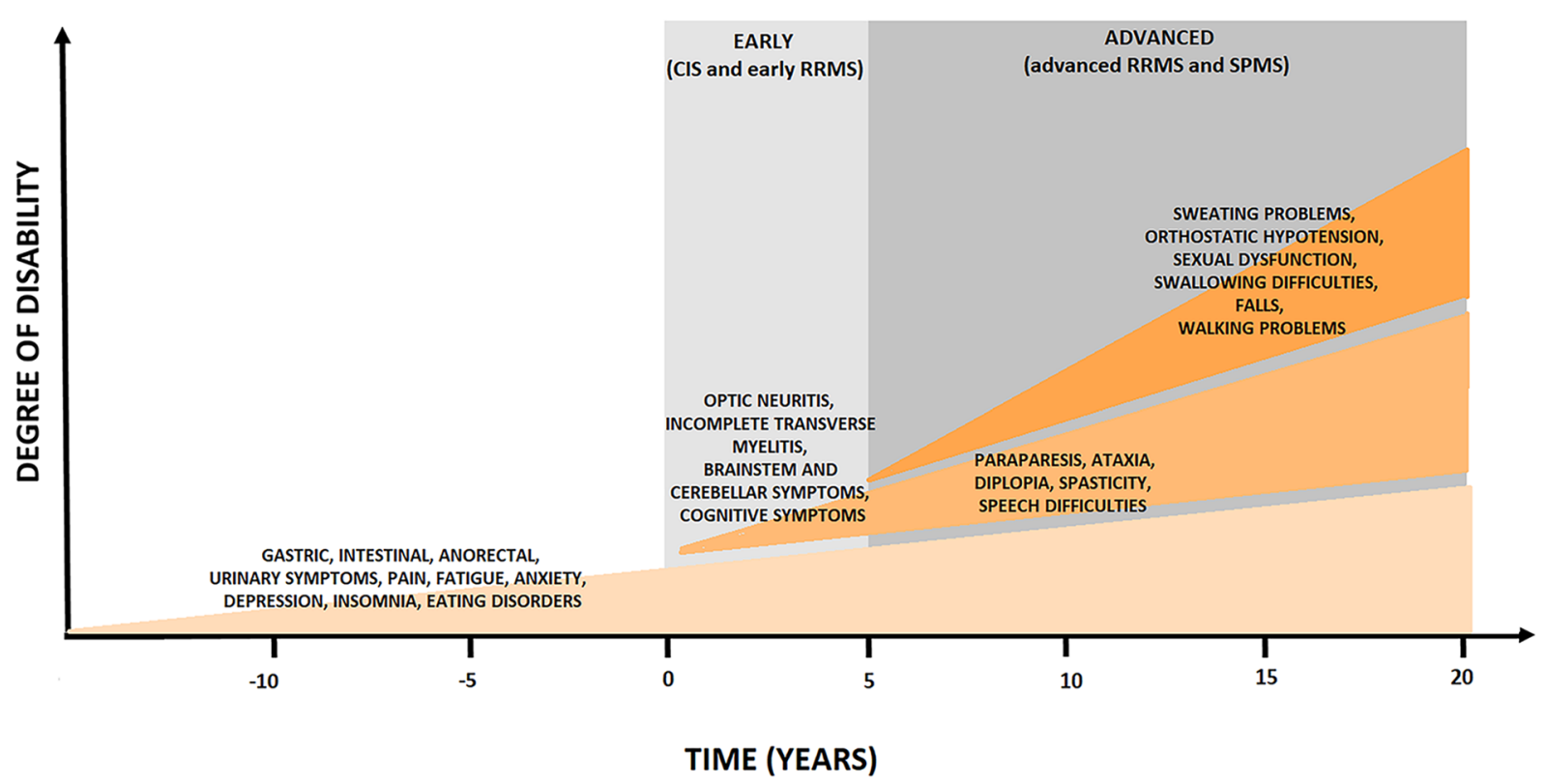

Fig. 1 Presentation of multiple sclerosis, from the prodromal or presymptomatic stage to secondary progressive multiple sclerosis. CIS clinically isolated syndrome, RRMS relapsing remitting multiple sclerosis, SPMS secondary progressive multiple sclerosis

while the disease progression (manifested by increasing neurological disability) is associated with parasympathetic nervous system dysfunction, with highest percentages of involvement seen in advanced progressive MS [9]. Furthermore, changes in expression of different receptors responsible for the communication between the autonomic and the immune systems may modulate inflammatory responses and thus have an influence on MS disease activity or progression [9].

Autonomic dysfunction, namely gastrointestinal, genitourinary, and cardiovascular abnormalities, is a key feature of the prodromal phase of other neurological disorders, such as Parkinson disease and other synucleinopathies [6]. Analog changes appear to occur in patients with MS, with sexual dysfunction appearing in later stages of disease and among patients with more advanced disability scores (Fig. 1) [6].

Moreover, autonomic symptoms are present and may also impact the disease course in MS. A recent study showed that the presence of autonomic symptoms increases the risk of a second demyelinating relapse after the diagnosis of clinically isolated syndrome by 2.7 -folds [10].

All these pieces of evidence indicate that the autonomic nervous system might be a key player in prodromal MS and in the development of clinically established MS. Studies ascertaining objective neurophysiologic and molecular markers of autonomic dysfunction in large populations of patients at risk of MS should confirm this.

Funding No funding was received.

\section{Compliance with ethical standards}

Conflict of interest The authors declare that they have no conflict of interest.

\section{References}

1. Magyari M, Sorensen PS (2019) The changing course of multiple sclerosis: rising incidence, change in geographic distribution, disease course, and prognosis. Curr Opin Neurol 32:320-326

2. Brown JWL, Coles A, Horakova D, Havrdova E, Izquierdo G, Prat A, Girard M, Duquette P, Trojano M, Lugaresi A, Bergamaschi R, Grammond P, Alroughani R, Hupperts R, McCombe P, Van Pesch V, Sola P, Ferraro D, Grand'Maison F, Terzi M, LechnerScott J, Flechter S, Slee M, Shaygannejad V, Pucci E, Granella F, Jokubaitis V, Willis M, Rice C, Scolding N, Wilkins A, Pearson OR, Ziemssen T, Hutchinson M, Harding K, Jones J, McGuigan C, Butzkueven H, Kalincik T, Robertson N, MSBase Study Group (2019) Association of initial disease-modifying therapy with later conversion to secondary progressive multiple sclerosis. JAMA 321:175-187

3. Yamout B, Al KM (2017) Radiologically isolated syndrome and multiple sclerosis. Mult Scler Relat Disord 17:234-237

4. Marrie RA, Yu N, Wei Y, Elliott L, Blanchard J (2013) High rates of physician services utilization at least five years before multiple sclerosis diagnosis. Mult Scler 19:1113-1119

5. Wijnands JMA, Kingwell E, Zhu F, Zhao Y, Högg T, Stadnyk K, Ekuma O, Lu X, Evans C, Fisk JD, Marrie RA, Tremlett H (2017) Health-care use before a first demyelinating event suggestive of a multiple sclerosis prodrome: a matched cohort study. Lancet Neurol 16:445-451

6. Disanto G, Zecca C, MacLachlan S, Sacco R, Handunnetthi L, Meier UC, Simpson A, McDonald L, Rossi A, Benkert P, Kuhle 
J, Ramagopalan SV, Gobbi C (2018) Prodromal symptoms of multiple sclerosis in primary care. Ann Neurol 83:1162-1173

7. Almeida MN, Silvernale C, Kuo B, Staller K (2019) Bowel symptoms predate the diagnosis among many patients with multiple sclerosis: a 14-year cohort study. Neurogastroenterol Motil 31:e13592

8. Bjornevik K, Munger KL, Cortese M, Barro C, Healy BC, Niebuhr DW, Scher AI, Kuhle J, Ascherio A (2019) Serum neurofilament light chain levels in patients with presymptomatic multiple sclerosis. JAMA Neurol. https://doi.org/10.1001/jamaneurol .2019 .3238

9. Habek M (2019) Immune and autonomic nervous system interactions in multiple sclerosis: clinical implications. Clin Auton Res 29:267-275

10. Krbot Skorić M, Crnošija L, Gabelić T, Barun B, Adamec I, Junaković A, Pavičić T, Ruška B, Habek M (2019) Autonomic symptom burden can predict disease activity in early multiple sclerosis. Mult Scler Relat Disord 28:250-255 